\title{
New Multiple-Target Tracking Strategy Using Domain Knowledge and Optimization
}

\author{
Runxiao Ding, Miao Yu, Member, IEEE, Hyondong Oh, and Wen-Hua Chen, Senior Member, IEEE
}

\begin{abstract}
This paper proposes an environment-dependent vehicle dynamic modeling approach considering interactions between the noisy control input of a dynamic model and the environment in order to make best use of domain knowledge. Based on this modeling, a new domain knowledge-aided moving horizon estimation (DMHE) method is proposed for ground moving target tracking. The proposed method incorporates different types of domain knowledge in the estimation process considering both environmental physical constraints and interaction behaviors between targets and the environment. Furthermore, in order to deal with a data association ambiguity problem of multiple-target tracking in a cluttered environment, the DMHE is combined with a multiple-hypothesis tracking structure. Numerical simulation results show that the proposed DMHE-based method and its extension could achieve better performance than traditional tracking methods which utilize no domain knowledge or simple physical constraint information only.
\end{abstract}

Index Terms-Domain knowledge, force-based model, moving horizon estimation (MHE), multiple-hypothesis tracking (MHT), multiple-target tracking (MTT).

\section{INTRODUCTION}

$\mathbf{T}$ RACKING multiple road users plays an important role in various applications such as surveillance, advanced driver assistance systems (ADASs), and autonomous vehicles. Many model-based state estimation methods have been proposed for target tracking. However, the majority of current methods assume an open field environment in which the tracked target(s) could move freely. This contradicts with the realistic scenario where the motion of the ground target(s) movement is often affected by its operational environment such as road and terrain. This information could be taken as domain knowledge

Manuscript received March 7, 2016; revised June 10, 2016; accepted September 20, 2016. Date of publication October 26, 2016; date of current version March 24, 2017. This work was supported in part by the U.K. Engineering and Physical Sciences Research Council under Grant $\mathrm{EP} / \mathrm{K} 014307 / 1$, and in part by the MOD University Defence Research Collaboration in Signal Processing and the Future Innovation Research Fund through the Ulsan National Institute of Science and Technology under Project 1.160086. The experimental dataset in this paper is provided on request by contacting: h.oh@unist.ac.kr. This paper was recommended by Associate Editor R. Roberts.

R. Ding, M. Yu, and W.-H. Chen are with the Department of Aeronautical and Automotive Engineering, Loughborough University, Loughborough, LE11 3TU, U.K.

H. Oh is with the School of Mechanical and Nuclear Engineering, Ulsan National Institute of Science and Technology, Ulsan 44919, South Korea (e-mail: h.oh@unist.ac.kr).

Color versions of one or more of the figures in this paper are available online at http://ieeexplore.ieee.org.

Digital Object Identifier 10.1109/TSMC.2016.2615188 and exploited in the development of tracking algorithms in order to enhance tracking quality and continuity.

The most apparent domain knowledge for ground vehicle tracking is the road constraint information such as the constrained region imposed by a road map. The studies on the road network-aided ground vehicle tracking have been reported in [1]-[6]. In these papers, the road network is taken as physical constraint information. Although comprehensive studies have been made for dealing with constraint information, limitations still exist. In particular, for a realistic tracking scenario, in addition to above physical road constraints, there are interactions between the target and its surrounding environment which need to be considered. For instance, the driver behaviors are affected by the surrounding environment and tend to obey the traffic rules. Drivers typically try to keep away from the road boundary while following the road/lane center and the speed limit. They also anticipate potential collision risks with incoming cars and make avoidance maneuvers whenever necessary.

An accurate dynamic model reflecting the aforementioned realistic movement of a vehicle is vital to obtain good tracking performance, especially when limited or even no measurements are available. However, most of the current vehicle dynamic models for target tracking [10], [11] predict the target's location from its past trajectory without fully taking into account the environmental interaction information. Recently, a social force model [12], [13] has been applied to model the interactions between pedestrians and environmental objects (building and walls) by using forces introduced by a potential field. These forces reflect different motion behaviors, for example, targets may be attracted to other objects or pushed away from them. However, the applications of the social force are limited to pedestrian tracking in the context of surveillance rather than vehicle tracking.

With this background, we propose a new vehicle dynamic modeling approach and its application to the multiple-target tracking (MTT) problem. The proposed modeling extends the traditional methods by incorporating the environmental information into the noisy control input of a dynamic model. The interaction between the target and the environment is modeled by virtual forces constructed by the target state, target dynamics and environment information. Compared with existing social force model used for pedestrian tracking [12], [13], the proposed model is more suitable for ground vehicle tracking involving much faster maneuvers as it utilizes the entire vehicle dynamic states (e.g., position and speed) and the 
predicted future position rather than using current position information only.

Among various target estimation algorithms [7], [8], the optimization-based moving horizon estimation (MHE) has a promising capability of being able to accommodate different types of constraints [9], [14], [25]. Thus, this paper proposes a domain knowledge-aided MHE method (DMHE) by using the aforementioned vehicle dynamic model, which incorporates both physical environmental constraints and interaction information into the tracking process in a comprehensive manner. The DMHE is further extended by combining with multiple-hypothesis tracking (MHT), denoted as the DMHEMHT, to deal with miss detection and false alarm considering a data association problem in a realistic MTT scenario. Note that, although miss detection and false alarms frequently occur in a cluttered environment, they have not been fully considered in most domain knowledge-aided tracking works [1]-[6]; only miss detection is considered in [2] and [3]. In the proposed DMHE-MHT strategy, tracking association ambiguity is handled by the MHT concept which associates measurements with corresponding targets. The association results are then applied for the DMHE optimization function construction.

This remaining part of this paper is structured as follows. The literature review on the associated problems in the domain knowledge-aided MTT field is presented in Section II. The domain knowledge dependent dynamic and measurement model are proposed in Section III. Section IV explains the DMHE-based target tracking algorithm, as well as its extension by combining it with the MHT to solve the MTT problem. In order to verify the benefit and efficiency of the proposed algorithm, numerical simulation results are presented in Section V. Finally, the conclusions are given in Section VI.

\section{Literature Review}

In this section, we review the MTT problem from its related three aspects: vehicle dynamic models, state estimation aided by domain knowledge (mainly the constraint information in the current works) and the data association.

\section{A. Vehicle Dynamic Models}

Various dynamic models can be generally divided into three categories: 1) macroscopic; 2) mesoscopic; and 3) microscopic models [28], [29]. In macroscopic models, the dynamics of the whole group of moving objects is described as an aggregate flow. Mesoscopic models determine the state of the system by the position or velocity distribution of each entity on the basis of aggregate relationships. Microscopic models refer to entities individually. In this case, the dynamics of every individual is considered by incorporating the social behavior of each target taking into account the interaction between the target and environmental moving/static objects. This paper focuses on incorporating the environmental information in a target tracking problem using the concept of the microscopic model. Examples of microscopic models include car-following model [28], cellular automata model [29], optimization-based models [30], and force-based models [12]. Among them, the force-based models have a great advantage of incorporating the environmental information as different sources of forces deterministically in a continuous model.

\section{B. Constrained State Estimation}

One of the effective approaches to solve a road-constrained MTT problem is to incorporate the constraint-related information into a standard filtering algorithm (i.e., state estimation process) as state constraints. Kalman filtering and its variations can be used to estimate the state of a target based on its dynamic and measurement models while considering limited constraint information. However, when the road state constraint types cannot fit easily into the structure of the Kalman filter (i.e., nonlinear and inequality constraints), they are often ignored or dealt with heuristically [25]. Recently, some other methods, for instance the constrained particle filters (PFs) and the constrained Gaussian mixture filters (see [8], [9], [31] for more details), are also developed based on optimization and truncation approaches. The majority of filters proposed to solve the constrained estimation problems focus on linear (in)equality or nonlinear equality constraints. A little research has been conducted on nonlinear inequality constraints so far. However, nonlinear inequality constraints (e.g., curved road boundary) are important and necessary for most tracking scenarios in ground vehicle tracking problems.

To address above issue, the MHE can be adopted, which proposed by Rao et al. [9] as a constrained state estimation method for nonlinear discrete-time systems. The basic strategy of the MHE is to reformulate the estimation problem as an optimization problem using a fixed-size estimation window. Theoretically, for a linear system without constraints and with a quadratic cost, the MHE becomes the same as the Kalman filter when an infinite horizon window is considered. This method has been widely used in chemical engineering. Other applications include hybrid systems, distributed, network systems, and large-scale systems. However, the implementation of the moving horizon approach in vehicle target tracking is still relatively an uncharted area.

Advantages of the MHE for target tracking are manifolds. First, since the method is based on optimization, road or similar constraints can be naturally handled by the MHE as additional (non)linear and/or (in)equality constraints. In addition to state constraints, the MHE is also able to incorporate constraints on the process and/or observation noises for modeling bounded disturbance or truncated distribution/density representing the influence of the operation environment on vehicle movement such as acceleration and deceleration. Another advantage of using the MHE in target tracking is that it always considers a certain number of latest measurements. Such feature is very meaningful in target tracking especially when targets are occluded by each other or static obstacles which leads to no reliable measurement during certain time steps. Simulation results in [7], [8], and [14] showed that MHE achieves the smallest estimation error for nonlinear systems and nonlinear constraints. It was also shown that the constrained MHE filter outperforms most of the other constrained algorithms (e.g., constrained PF, constrained 
unscented Kalman filter, etc.) in terms of estimation error while keeping an acceptable computational time for target tracking applications with a relatively simple implementation process.

\section{Data Association}

Different techniques can be used to deal with the miss detections and false alarms for MTT data association problems; for example, nearest neighbor standard filter [15], global nearest neighbor approach [16], joint probabilistic data association [17], MHT algorithm [18], and finite set statistics [19]. Among them, the MHT is a complex approach that considers data association across multiple scans and multiple hypotheses. The MHT essentially keeps a set of multiple hypotheses, and the assignment ambiguity will be resolved in future when subsequent new observations are arrived. Hard decisions are not made until they are needed with sufficient information rather than just the current data frame; thus possible error association could be corrected when more evidences are obtained. Such features along with the dramatic increases in computational capabilities have made the MHT a preferred data association method for modern tracking systems [20].

\section{ENVIRONMENTAL INFORMATION-AIDED Dynamic and Measurement Model}

\section{A. Environmental Information-Aided Dynamic Model}

First, let us review the general dynamic model for target tracking problem

$$
x_{k+1}=f\left(x_{k}\right)+\omega_{\mathrm{k}}
$$

where $x_{k}$ represents the state vector, which usually includes the position and velocity for tracking problem. $\omega_{\mathrm{k}}$ is generally known as the process noise and more specifically considered as noisy acceleration components that controls the dynamic evolution of $\boldsymbol{x}_{\boldsymbol{k}}$ and follows a certain type of distribution to represent uncertainty of a driver's behavior. $f(\cdot)$ represents the system dynamic function which reflects a desired target dynamic type representing the state transition between consecutive time steps. According to [10], in most of the target tracking problems, the control term $\omega_{\mathrm{k}}$ is modeled as a Gaussian distribution with zero mean and constant covariance matrix representing target movement uncertainty irrespective to the surrounding environment. However, in realistic tracking scenarios, targets' movements are affected by the surrounding environment (e.g., road boundary, road centerline or speed limit). In other words, the vehicle noisy control input $\omega_{\mathrm{k}}$ from uncertain driver behaviors is related with the environment.

Therefore, this section proposes a new vehicle dynamic modeling approach which incorporates environmental information into the vehicle control input, inspired by the social force model [12], [21]. In the original social force model as in [12] and [21], pedestrians are assumed moving with low and constant velocity in a short time interval and force is considered to be only related to the relative distance between pedestrian's current position and other environmental objects.

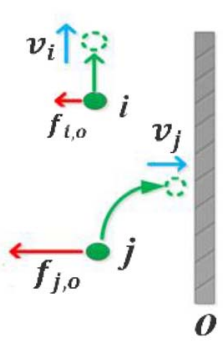

(a)

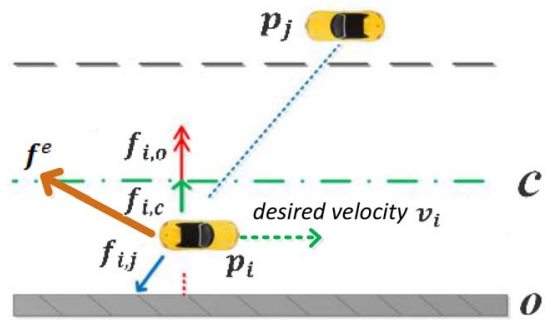

(b)
Fig. 1. Influence of the environment on a moving target by forces. (a) Different repulsive forces $f_{i, o}$ and $f_{j, o}$ on objects $i$ and $j$ with different dynamics between $T=t$ (when objects position are marked as green circles) and $T=t+\Delta t$ (where objects position are marked as dashed circles). (b) $p_{i}$ receives interaction force $f_{i, j}$ from another vehicle, attractive force $f_{i, c}$ from the centerline and repulsive force $f_{i, o}$ from the road boundary.

Compared with the human tracking scenario, our problem exhibits much more complex vehicle movements with high velocity. In this case, the force (control) term needs to consider not only position but velocity information and the desired dynamics of the vehicle. As illustrated in Fig. 1(a), the object $j$ is assumed to perform a turning maneuver should receive a higher repulsive force than the object $i$ because it will get closer to the boundary. Besides, forces should also relate to the magnitude of the velocity; for instance, if the velocity $\boldsymbol{v}_{j}$ of the object $j$ toward the road boundary becomes larger, a larger repulsive force should be imposed on the object.

In the proposed dynamic model, both repulsive and attractive effects from the environment are considered where the repulsive (or attractive) force is modeled as a monotonously decreasing (or increasing) exponential function. According to the current state $\boldsymbol{x}_{\boldsymbol{k}}$ (including both position and velocity states) of the vehicle $i$, the predicted position $x_{i}^{\text {predict }}$ is first calculated from the dynamic model determined by $f\left(\boldsymbol{x}_{\boldsymbol{k}}\right)$. In this way, the entire state and dynamic model information are incorporated. Then, the relative Euclidean distance $d_{i j}^{\text {prediction }}$ between $x_{i}^{\text {predict }}$ and position of the object $j$ (e.g., road boundary, road centerline or other vehicles) is estimated. The repulsive/attractive force between target $i$ and object $j$ can then be represented as

$$
\begin{aligned}
& \boldsymbol{f}_{i, j}^{\text {repulsive }}=A \cdot \exp \left(\frac{-d_{i j}^{\text {prediction }}}{B}\right) \boldsymbol{n}_{j i} \\
& \boldsymbol{f}_{i, j}^{\text {attractive }}=A \cdot\left(1-\exp \left(\frac{-d_{i j}^{\text {prediction }}}{B}\right)\right) \boldsymbol{n}_{i j}
\end{aligned}
$$

where $A$ and $B$ are positive constants representing the magnitude and range of the force, respectively. $\boldsymbol{n}_{i j}$ is the normalized vector pointing form $i$ to $j$.

As shown in Fig. 1(b), it is assumed that there exist different forces acting on ego vehicle $i$ generated by the surrounding environmental objects, such as the repulsive force $f_{i, \boldsymbol{o}}$ from road boundary $o$, attractive force $f_{i, c}$ to the centerline $c$ and the repulsive force $f_{i, j}$ from another moving vehicle $j$ to avoid a collision.

These forces are summed to a net environmental force $f^{e}$ acting on the vehicle $i$, which can be incorporated into the 
dynamic model (1) as

$$
\boldsymbol{x}_{k+1}=f\left(\boldsymbol{x}_{k}\right)+I\left(\boldsymbol{a}_{k}^{e}\right)+\boldsymbol{\omega}_{k}
$$

where $\boldsymbol{a}_{k}^{e}=\left(\boldsymbol{f}_{k}^{e} / m\right)$ represents the acceleration introduced by the environmental force. $I\left(\boldsymbol{a}_{k}^{e}\right)$ is the function representing the influence of the acceleration $\boldsymbol{a}_{k}^{e}$ on the vehicle dynamic model, which has different forms according to different representations of (1).

\section{B. Environmental Information-Aided Measurement Model}

For the model based tracking problem, usually measurements are associated with a measurement model which can be generally represented as

$$
\boldsymbol{y}_{k}=h\left(\boldsymbol{x}_{k}\right)+\boldsymbol{v}_{k}
$$

where $\boldsymbol{y}_{k}$ is a measurement vector, $h\left(\boldsymbol{x}_{k}\right)$ is the measurement function, and $\boldsymbol{v}_{k}$ is zero mean Gaussian noise of the measurement with the covariance $R$.

We assume that the ground vehicles only move within the road network region. This matches with a realistic scenario where road boundaries are considered as physical constrains and all drivers are supposed to move within the constraint region. Due to the limited tracking sensor's capability, the received measurements usually contain noises as in (5), which make them not always stay on the road network and far away from the ground truth values. Such noisy measurements are usually known as false alarms in MTT which make data association process really difficult with tracking ambiguity problems. To this end, a preprocessing approach is used in this paper to project the raw measurements onto the constrained surface (road network) at each time step.

Assuming that target vehicles are traveling on a linear road following the center line, the raw measurement data $\boldsymbol{y}_{k}$ could then be projected by the following linear equality constraint as:

$$
D \widetilde{y}_{k}=\boldsymbol{d}_{k}
$$

where $D$ is a full-rank constraint matrix and $\boldsymbol{d}_{k}$ is the constraint vector. $\widetilde{\boldsymbol{y}}_{k}$ is the projected (constrained) Cartesian measurement. Following [25], the expression of deriving constrained measurement $\tilde{\boldsymbol{y}}_{k}$ by directly projecting the unconstrained Cartesian coordinate measurement $\boldsymbol{y}_{k}$ onto the constraint surface is by solving the problem

$$
\min _{\widetilde{\boldsymbol{y}}_{k}}\left(\tilde{\boldsymbol{y}}_{k}-\boldsymbol{y}_{k}\right) W\left(\widetilde{\boldsymbol{y}}_{k}-\boldsymbol{y}_{k}\right), \quad \text { s.t. } \quad D \widetilde{\boldsymbol{y}}_{k}=\boldsymbol{d}_{k}
$$

where $W$ is a symmetric positive definite weighting matrix. In this paper, it is chosen as $W=R$ following the mean square method, where $R$ is a measurement error covariance matrix of the original measurements. The solution of this problem is then given by

$$
\tilde{\boldsymbol{y}}_{k}=\boldsymbol{y}_{k}-R^{-\mathbf{1}} D^{\boldsymbol{T}}\left(D R^{-\mathbf{1}} D^{\boldsymbol{T}}\right)^{-1}\left(D \boldsymbol{y}_{k}-\boldsymbol{d}_{k}\right) .
$$

According to [25], the projected measurement error covariance matrix $\tilde{R}$ can be expressed as

$$
\begin{aligned}
\tilde{R}=\left(I-R^{-1} D^{T}\left(D R^{-1} D^{T}\right)^{-1} D\right) R & \\
& \left(I-R^{-1} D^{T}\left(D R^{-1} D^{T}\right)^{-1} D\right)^{T} .
\end{aligned}
$$

In this way, the measurement model is modified as

$$
\tilde{\boldsymbol{y}}_{k}=\left[\begin{array}{l}
x_{k} \\
y_{k}
\end{array}\right]+\widetilde{\boldsymbol{v}}_{k}
$$

where $\tilde{\boldsymbol{y}}_{k}$ is the projected measurement, $\left[\begin{array}{l}x_{k} \\ y_{k}\end{array}\right]$ represents the target position, and $\widetilde{\boldsymbol{v}}_{k}$ is the measurement noise for the projected measurement with zeros mean and covariance $\tilde{R}$.

For nonlinear road constraint $g\left(\widetilde{\boldsymbol{y}}_{k}\right)=\boldsymbol{d}_{k}$, the development of the constrained measurement (10) as given above is still valid with a linearization process. We can perform a first order Taylor series expansion of the constraint equation around $\tilde{\boldsymbol{y}}_{k}$ to obtain

$$
g\left(\widetilde{\boldsymbol{y}}_{k}\right)=\boldsymbol{d}_{k} \approx g\left(\boldsymbol{y}_{k}\right)+g^{\prime}\left(\boldsymbol{y}_{k}\right)\left(\widetilde{\boldsymbol{y}}_{k}-\boldsymbol{y}_{k}\right)
$$

which indicates that

$$
g^{\prime}\left(\boldsymbol{y}_{k}\right) \widetilde{\boldsymbol{y}}_{k} \approx \boldsymbol{d}_{k}-g\left(\boldsymbol{y}_{k}\right)+g^{\prime}\left(\boldsymbol{y}_{k}\right) \boldsymbol{y}_{k} .
$$

We now have an approximated nonlinear constraint that is equivalent to the linear constraint $D \widetilde{\boldsymbol{y}}_{k}=\boldsymbol{d}_{k}$, where $D$ is replaced with $g^{\prime}\left(\boldsymbol{y}_{k}\right)$ and $\boldsymbol{d}_{k}$ is replaced with $\boldsymbol{d}_{k}-g\left(\boldsymbol{y}_{k}\right)+$ $g^{\prime}\left(\boldsymbol{y}_{k}\right) \boldsymbol{y}_{k}$.

In a realistic ground vehicle tracking scenario, each vehicle may undergo different regions with different environmental conditions. Thus, one single model might not be able to accurately describe various movement types in different environmental conditions. Considering that multiple-state models are involved, before performing the DMHE-MHT algorithm, it is required to determine the particular state model which best fits to describe the vehicle movement. To this end, this paper adopts the Bayesian inference framework which utilizes the posterior model probability to decide the appropriate state model at each time step. The implementation of the Bayesian inference is done by interactive multiple-model particle filtering (IMMPF) method among others. The details for the IMMPF can be found in [26].

\section{MHE-BASEd TARget TRACKing With ENVIRONMENTAL INFORMATION}

Based on the domain knowledge-aided dynamic modeling and measurements as mentioned in the previous section, the MHE based optimization scheme is applied for the state estimation, which is detailed as follows.

\section{A. General Moving Horizon Estimation}

By the original state model (1) and measurement model (5) without considering any environmental information, the standard MHE is defined as an optimization problem by the following form [9]:

$$
\begin{array}{r}
\min _{\left\{x_{k-N},\left\{\omega_{j}\right\}_{j=k-N}^{k-1}\right\}} \sum_{j=k-N}^{k-1}\left(\left\|x_{j}-f\left(x_{j-1}\right)\right\|_{Q^{-1}}^{2}\right. \\
\left.+\left\|\boldsymbol{y}_{j}-h\left(x_{j}\right)\right\|_{R^{-1}}^{2}\right)+\Gamma_{k-N}\left(\boldsymbol{x}_{k-N}\right)
\end{array}
$$

for $\left\{\boldsymbol{x}_{k-N}, \ldots, \boldsymbol{x}_{k}\right\} \in C_{x}$ 
where $C_{x}$ represents the constrained region which represents the feasible road region determined by the road network in the ground vehicle tracking problem. $\left\{\boldsymbol{x}_{k-N: k}\right\}$ is the ensemble of states from time instance $k-N$ to $k$ which solves the quadratic programming problem (13) while giving the optimal estimate solution. $N$ is a moving horizon length which is chosen to give a tradeoff between the estimation accuracy and the computational cost. $\Gamma_{k-N}\left(\boldsymbol{x}_{k-N}\right)=-\log \left(\mathrm{p}\left(\boldsymbol{x}_{k-N} \mid Y_{0: k-N-1}\right)\right)$ represents an arrival cost which plays an important role in summarizing the effect of the past measurement as a priori information on the initial state $\boldsymbol{x}_{k-N}$

$$
\Gamma_{k-N}\left(\boldsymbol{x}_{k-N}\right) \approx\left\|\boldsymbol{x}_{k-N}-\widehat{\boldsymbol{x}}_{k-N}^{m h}\right\|_{P_{k-N}^{-1}}^{2}
$$

where $\widehat{\boldsymbol{x}}_{k-N}^{m h}$ and $P_{k-N}$ represents the previous moving horizon state estimate and covariance at $k-N$, respectively. The unconstrained extended Kalman filter (EKF) [9] is adopted as the approximate method for calculating the arrival cost error covariance matrix $P_{k+1}$ by using the following update rule:

$$
\begin{aligned}
P_{k-N}=Q+F P_{k-N-1} F^{T}-F P_{k-N-1} H^{T} & \\
& \times\left(R+H P_{k-N-1} H^{T}\right)^{-1} H P_{k-N-1} F^{T}
\end{aligned}
$$

where $F$ and $H$ represent the Jacobian matrix of the function $f\left(\boldsymbol{x}_{k}\right)$ and $h\left(\boldsymbol{x}_{k}\right)$, respectively.

The state estimate of the MHE optimization function (13) at time $k$ is denoted as $\boldsymbol{x}\left(k ; \widehat{\boldsymbol{x}}_{k-N}^{*},\left\{\widehat{\boldsymbol{\omega}}_{j}^{*}\right\}_{j=k-N}^{k-1}\right)$, including the optimized initial state $\widehat{\boldsymbol{x}}_{k-N}^{*}$ and the optimized process noise sequence $\left\{\widehat{\boldsymbol{\omega}}_{j}^{*}\right\}_{j=k-N}^{k-1}$. The optimized estimated state $\widehat{\boldsymbol{x}}_{k}^{*}$ at time instance $k$ considering a linear dynamic system can be expressed as

$$
\begin{aligned}
\widehat{\boldsymbol{x}}_{k}^{*} & =\boldsymbol{x}\left(k ; \widehat{\boldsymbol{x}}_{k-N}^{*},\left\{\widehat{\boldsymbol{\omega}}_{j}^{*}\right\}_{j=k-N}^{k-1}\right) \\
& =F^{k} \widehat{\boldsymbol{x}}_{k-N}^{*}+\sum_{j=k-N}^{k-1} F^{k-j-1} \widehat{\boldsymbol{\omega}}_{j}^{*} .
\end{aligned}
$$

\section{B. Domain Knowledge-Aided Moving Horizon Estimation}

Although the aforementioned MHE method could incorporate the constraint information for the state estimation, it cannot exploit the environmental information in a comprehensive way.

1) The interaction between the target and surrounding environment (e.g., a vehicle keeps away from static/moving environmental objects, such as road boundary, another vehicle, etc.) is not considered in the original MHE framework.

2) Domain knowledge is not considered in the measurement model.

To this end, a new framework of the MHE which fully exploits the domain knowledge (denoted as DMHE for short) is proposed. Both the proposed state model (4), which considers the interaction information and the projected measurement model (10) are used to construct a new MHE optimization function as

$$
\begin{aligned}
\min _{\left\{\boldsymbol{x}_{k-N},\left\{\boldsymbol{\omega}_{j}\right\}_{j=k-N}^{k-1}\right\}} \sum_{j=k-N+1}^{k}( & \left\|\boldsymbol{x}_{j}-f\left(\boldsymbol{x}_{j-1}\right)-I\left(\boldsymbol{a}_{j}^{e}\right)\right\|_{Q^{-1}}^{2} \\
& \left.+\left\|\tilde{\boldsymbol{y}}_{j}-h\left(\boldsymbol{x}_{j}\right)\right\|_{\widetilde{\boldsymbol{R}}^{-1}}^{2}\right)+\Gamma_{k-N}\left(\boldsymbol{x}_{k-N}\right)
\end{aligned}
$$

for $\left\{x_{k-N}, \ldots, x_{k}\right\} \in C_{x}$.

Compared with the MHE function in (13), besides the road constraint based information the domain knowledge is better exploited from two aspects.

1) A new $I\left(\boldsymbol{a}_{j}^{e}\right)$ term is introduced, which is related to the environmental force modeling the interaction as mentioned previously. In this way, the interaction information is considered in the MHE process.

2) The projected measurements $\widetilde{y}_{k}$ and associated error covariance $\tilde{R}$ are exploited to better model measurement information.

By solving the DMHE cost function (17), the optimized estimated state at time $k$ can be obtained as

$$
\begin{array}{r}
\widehat{\boldsymbol{x}}_{k}^{*}:=\boldsymbol{x}\left(k ; \widehat{\boldsymbol{x}}_{k-N}^{*},\left\{\widehat{\boldsymbol{\omega}}_{j}^{*}\right\}_{j=k-N}^{k-1},\left\{f_{j}^{e, *}\right\}_{j=k-N}^{k-1}\right)=F^{k} \widehat{\boldsymbol{x}}_{k-N}^{*} \\
+\sum_{j=k-N}^{k-1} F^{k-j-1}\left(\widehat{\boldsymbol{\omega}}_{j}^{*}+I\left(\boldsymbol{a}_{j}^{e, *}\right)\right) .
\end{array}
$$

Note that, $\boldsymbol{a}_{j}^{e, *}$ is a function of $\widehat{\boldsymbol{x}}_{k-N}^{*}$ and $\left\{\widehat{\boldsymbol{\omega}}_{j}^{*}\right\}_{j=k-N}^{k-1}$, according to the force terms defined in (2) and (3). The covariance required for the arrival cost computation as in (15) is modified by considering the influence of the term $I\left(\boldsymbol{a}_{k}^{e}\right)$ at time instance $k$ by

$$
\begin{aligned}
P_{k-N}= & Q+\left(F+\nabla_{\widehat{\boldsymbol{x}}_{k-N-1}^{*}} I\left(\boldsymbol{a}_{k-N-1}^{e}\right)\right) P_{k-N-1} \\
& \times\left(F+\nabla_{\widehat{\boldsymbol{x}}_{k-N-1}^{*}} I\left(\boldsymbol{a}_{k-N-1}^{e}\right)\right)^{T} \\
& -\left(F+\nabla_{\widehat{\boldsymbol{x}}_{k-N-1}^{*}} I\left(\boldsymbol{a}_{k-N-1}^{e}\right)\right) P_{k-N-1} H^{T} \\
& \times\left(R+H P_{k-N-1} H^{T}\right)^{-1} H P_{k-N-1} \\
& \times\left(F+\nabla_{\widehat{\boldsymbol{x}}_{k-N-1}^{*}} I\left(\boldsymbol{a}_{k-N-1}^{e}\right)\right)^{T}
\end{aligned}
$$

where $\nabla_{\widehat{\boldsymbol{x}}_{k}^{*}} I\left(\boldsymbol{a}_{k}^{e}\right)$ represents the gradient of the term $I\left(\boldsymbol{a}_{k}^{e}\right)$ with respect to $\widehat{x}_{k}^{*}$ at time instance $k$.

\section{DMHE-Based Multiple-Hypothesis Tracking}

The proposed DMHE algorithm is further extended to address the data association problem by incorporating it into an MHT structure, which constructs a DMHE-MHT framework for MTT in a more complicated scenario with both miss detections and false alarms. Comparing with other data association algorithms, the MHT has the advantages of being able to deal with track creation, confirmation, occlusion and deletion in a probabilistically consistent way and keeping a multiple number of past hypotheses between consecutive time steps [22]. In this combined DMHE-MHT strategy, tracking association ambiguity is handled by MHT data association. After measurements are associated with proper targets, vehicle states are then estimated by the DMHE algorithms by exploiting the 


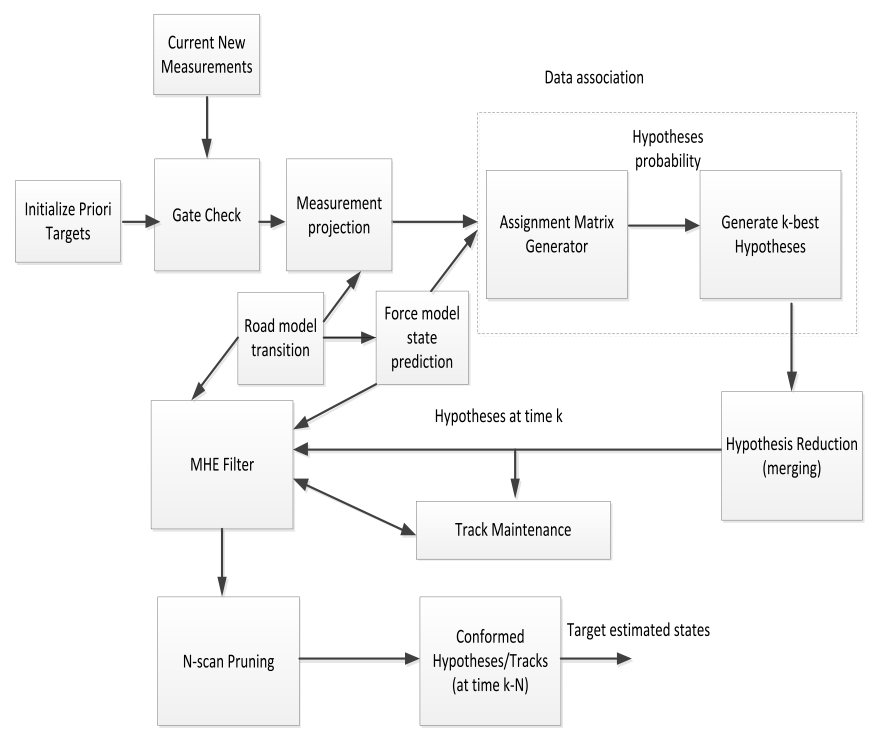

Fig. 2. Flow diagram of DMHE-MHT algorithm.

domain knowledge. The block diagram of the DMHE-MHT algorithm is presented in Fig. 2.

Initially, let $Y_{k}=\left\{y_{i}^{k}\right\}_{i=1}^{m_{k}}$ denote the set of $m_{k}$ measurements received at time $k$. Each measurement has three possible hypotheses: 1) the measurement starts a new target; 2) the measurement is a false alarm; and 3) the measurement belongs to an existing target. The procedures of the DMHE-MHT are divided into the following steps.

1) Gate Check: First, the distance between the predicted (prior) target position and the current measurements is calculated. The prediction of target position is done by the Kalman filter time update, and the distance is defined as the Mahalanobis distance as

$$
\left(\boldsymbol{y}_{m}^{k}-\overline{\boldsymbol{y}}_{k / k-1}\right)^{T} S_{k / k-1}\left(\boldsymbol{y}_{m}^{k}-\overline{\boldsymbol{y}}_{k / k-1}\right)
$$

where $\boldsymbol{y}_{m}^{k}$ is the $m$ th measurement at time $k, \overline{\boldsymbol{y}}_{k / k-1}$ is the predicted target position, and $S_{k / k-1}$ is the covariance of innovation vector corresponding to the position. Note that the predicted position is calculated by the forced-aided model (4) considering environmental interaction information. Gating is a matrix of binary values which indicates the maximum possible distance between measurements and targets. The measurements whose Mahalanobis distances with particular targets are smaller than a particular threshold are used for the further data association.

2) Measurement Projection: After the gating process, the candidate measurements within the gating region are projected to the road using the process described in Section IV considering road constraints. The state dependent road model transition process is used to determine on which road the target is moving. $\widetilde{\boldsymbol{y}}$ and $\widetilde{\boldsymbol{R}}$ are calculated for the data association process.

3) State Prediction: After determining on which road the target is moving, the corresponding target-environment interaction force is calculated. The force-based state dynamic prediction is then calculated which will be used in both data association and the MHE process.
4) Data Association: The FDHE-MHT implements a similar data association process as the Reids algorithm [18] with the projected measurement $\widetilde{\boldsymbol{y}}$, the constrained measurement error covariance $\widetilde{\boldsymbol{R}}$ and the forced-based state prediction. The assignment matrix is generated to represent all possible targetto-measurement associations. Then, each new hypothesis contains a set of potential target-to-measurement assignments, leading to an exhaustive process of enumerating all the possible assignment combinations. To address this issue, the Murty's algorithm [24] is used to find the $k$-best assignment and new hypotheses generated from each parent hypothesis. To further reduce the computational cost, a merging algorithm is also implemented to prevent hypotheses from being considered if the ratio of their probability to the best hypothesis becomes too small.

5) Target Maintenance: In ground target tracking scenarios, vehicles may enter or leave the region of interest during the tracking process. Moreover, occlusion or miss detection is also possible when a vehicle is hidden behind other objects. Based on the data association results, we implement target maintenance to identify targets which are entering, staying or leaving the tracking scene, by considering three possible statuses for a set of targets: 1) target initiation; 2) confirmation/deletion; and 3) maintenance. Table I summarizes the target maintenance procedure.

1) Target Initiation: If the measurement is associated with a new target, then the new target hypothesis appears in the current $k$-best hypotheses. A target lifetime index is added to the target with value 1 .

2) Target Confirmation/Deletion: The new target is confirmed only if the detected target appears along the same track over a consecutive iteration of $\mathrm{Ct}$ (confirmation threshold) times. The lifetime index is accumulated by 1 whenever the tentative target is detected and will become $\mathrm{Ct}$ when confirmed. On the contrary, the lifetime index for any existing target is reduced by 1 whenever the target is not associated with the current measurement and will be permanently deleted from the target list when the lifetime is 0 .

3) Target Maintenance: The confirmed target may be temporally occluded or undetected by the sensor without measurements being associated. For this situation, the track is updated according to the predicted position of the target last associated states.

6) DMHE Filter: The details about implementing the DMHE for constrained target tracking based on associated measurements have been discussed in the previous section. Note that, in the original MHT, the "filter" process is based on the KF consisting of: time update (i.e., prediction) and measurement update. However, these two steps are combined in the DMHE and solved concurrently by the optimization process. The state estimation is determined online by solving a state estimation problem for a finite horizon window.

7) $\mathrm{N}$-Scan Pruning: An $N$-scan pruning technique [24] is applied in the MHT structure to control the complexity of the algorithm. The growing number of hypotheses is controlled by $N$-scan pruning technique by keeping only the ones with the largest probability values (detailed as in [24]), while other 
TABLE I

High-LEVEl Logic FOR DMHE-MHT TARGET MAINTENANCE

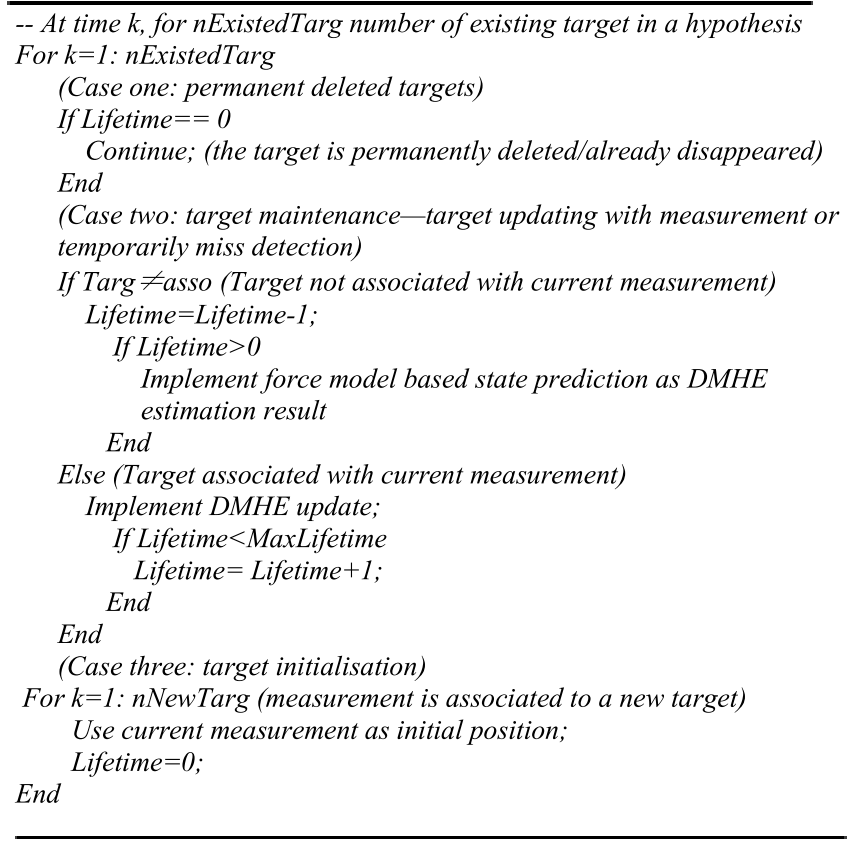

hypotheses with low probability are deleted after $N$-scan pruning. In the DMHE-MHT, the number of $N$ scans is chosen as the same value for the horizon length in the MHE.

\section{Numerical Simulation Results}

In this section, two simulation examples are presented in the context of ground vehicle tracking. The first example is single target tracking, aiming at illustrating the proposed DMHE with both linear and nonlinear inequality road constraint. The second one is a complex multiple vehicle tracking scenario incorporating road inequality constraints from real-world map data for the DMHE-MHT.

\section{A. Single Target Tracking}

The proposed DMHE algorithm is evaluated by single target tracking scenario for both linear (position) and nonlinear (bearing/range) measurement models with road boundary constraints. The first one is a linear trajectory, considering a single carriageway with road width of $4 \mathrm{~m}$ and an angle of $45^{\circ}$ anticlockwise to the horizontal axis. The vehicle dynamics is described by a constant velocity model with the noisy acceleration

$$
\boldsymbol{x}_{k+1}=\left[\begin{array}{cccc}
1 & 0 & T & 0 \\
0 & 1 & 0 & T \\
0 & 0 & 1 & 0 \\
0 & 0 & 0 & 1
\end{array}\right] \boldsymbol{x}_{k}+\left[\begin{array}{cc}
T 2 / 2 & 0 \\
0 & T 2 / 2 \\
T & 0 \\
0 & T
\end{array}\right] \boldsymbol{\omega}_{k}
$$

where the state vector $\boldsymbol{x}_{k}=\left[x_{1, k}, x_{2, k}, \dot{x}_{1, k}, \dot{x}_{2, k}\right]^{T}$ consists of the vehicle position and velocity in $x$ and $y$ directions, and $T=1$ is the sampling interval, $\omega_{k}$ is a $2-\mathrm{D}$ Gaussian process noise with zero mean and covariance matrix $Q=\operatorname{diag}\{5,2\}$ in a local coordinate, where diag\{.\} represents a diagonal matrix. This covariance represents higher motion uncertainty along the center line direction and smaller uncertainty orthogonal to the road. The vehicle measurement model is a linear matrix in $x$ and $y$ position with a Gaussian measurement noise $\boldsymbol{v}_{k}$ and covariance matrix $R=\operatorname{diag}\{20 / \sqrt{2}, 20 / \sqrt{2}\}$ in a global Cartesian coordinate as

$$
z_{k}=\left[\begin{array}{llll}
1 & 0 & 0 & 0 \\
0 & 1 & 0 & 0
\end{array}\right] \boldsymbol{x}_{k}+\boldsymbol{v}_{\boldsymbol{k}}
$$

The vehicle has a center line direction velocity of $10 \mathrm{~m} / \mathrm{s}$ with no initial lateral velocity, and the initial state is $\boldsymbol{x}_{0}=$ $[0,1502.83,7.0711,-7.0711]^{T}$.

The movement of the target is constrained by road boundaries and supposed to follow the center line of the road. Different environmental forces are considered including lateral forces orthogonal to the road as follows.

1) Repulsive force generated from lower road boundary

$$
\boldsymbol{f}_{i, j}^{\mathrm{rep} 1}=A \cdot \exp \left(\frac{-d_{\mathrm{lb}}}{B}\right) \boldsymbol{n}_{j i} \text {. }
$$

2) Repulsive force generated from upper road boundary

$$
\boldsymbol{f}_{i, j}^{\mathrm{rep} 2}=A \cdot \exp \left(\frac{-d_{\mathrm{ub}}}{B}\right) \boldsymbol{n}_{j i} .
$$

3) Attractive force to center line of the road

$$
\boldsymbol{f}_{i, j}^{\text {att }}=A \cdot\left(1-\exp \left(\frac{-d_{\text {center }}}{B}\right)\right) \boldsymbol{n}_{i j}
$$

where $i$ and $j$ represents the target and the environment (road boundary, center line, and speed limit where applicable), respectively. $d_{\mathrm{lb}}$ and $d_{\mathrm{ub}}$ represent the Euclidean distance between lower and upper boundary of the road and the predicted vehicle position $x_{i}^{\text {predict }}$ calculated from the dynamic model (11) based on the current location, respectively. Similarly, $d_{\text {center }}$ represents the distance between center line and predicted vehicle position. Note that the closer (further) the vehicle gets to the road boundaries (away from the center line), the larger the repulsive (attractive) force will be generated.

Besides the above lateral forces, a velocity-based breaking (repulsive) force is also considered along the center line direction so as to present the road speed limitation

$$
\boldsymbol{f}_{i, j}^{\mathrm{rep} 3}=-A \cdot \exp \left(\frac{-\left(v_{\text {limit }}-v_{\text {heading }}\right)}{B}\right) \boldsymbol{v}
$$

where $v_{\text {heading }}=\sqrt{\dot{x}_{k}^{2}+\dot{y}_{k}^{2}}$ is the speed of the vehicle toward heading direction. The speed limitation $v_{\text {limit }}$ is defined as a valid speed interval around a specific speed value. And $v$ represents a unit velocity vector. Although the relative speed difference can be either positive or negative, the repulsive effect is much larger when the vehicle exceeds the speed limit, as illustrated in Fig. 3.

To evaluate the performance, four different tracking models are compared.

1) General MHE without considering any environmental information (MHE).

2) Force-based MHE without considering physical constraints (FMHE).

3) General MHE with inequality physical constraints (road boundaries) (CMHE).

4) The proposed DMHE approach. 


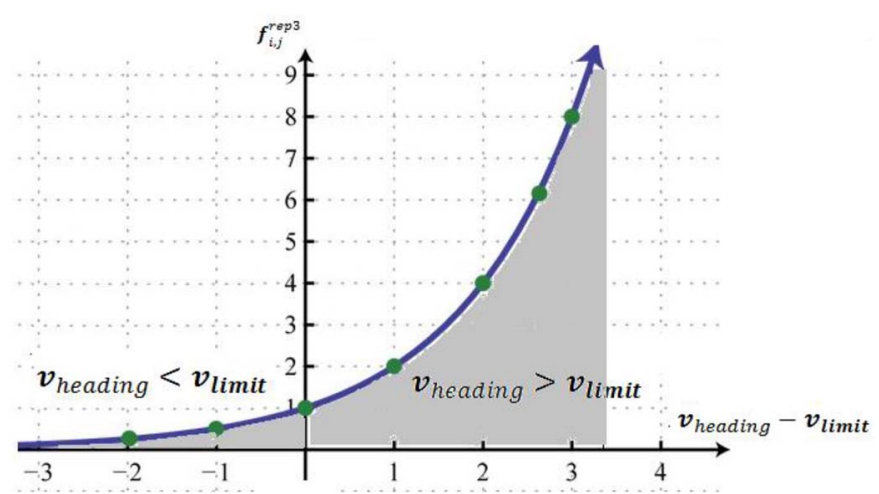

Fig. 3. Force generated from the speed limitation.

TABLE II

ESTIMATION PERFORMANCE COMPARISON OF MHE, FMHE, CMHE, AND DMHE

\begin{tabular}{|c|c|c|c|c|}
\hline RMSE (m) & MHE & FMHE & CMHE & DMHE \\
\hline Position (m) & 2.6506 & 2.4206 & 2.4877 & 2.3216 \\
\hline Centre line direction (m) & 3.1298 & 2.9606 & 3.1181 & 2.9414 \\
\hline Orthogonal direction (m) & 2.8932 & 2.5160 & 1.6295 & 1.4588 \\
\hline
\end{tabular}

In Table II, the performance of different models is compared in terms of root mean-square error (RMSE) in three different aspects: 1) position RMSE; 2) center line tangential direction position RMSE; and 3) orthogonal position RMSE to the road with a horizon length of $N=4$. It is shown that road physical constraint is of great importance when comparing the CMHE with the MHE and the DMHE with the DMHE, especially in orthogonal direction where road boundary is considered. In addition to physical constraints, environmental forces further improve the estimation accuracy. Both FMHE and DMHE have shown a significant improvement for target's position estimate compared with their relative MHE and CMHE.

In the second scenario, a vehicle is simulated to move along the quarter of a circular road with an angular velocity of $0.1 \mathrm{rad} / \mathrm{s}$ along the road centerline for $15 \mathrm{~s}$. Small noises are added to the simulated vehicle position to represent the disturbance of the vehicle movement. The road has a width of $4 \mathrm{~m}$ and is defined by two arc boundaries of $r_{1}=96 \mathrm{~m}$ and $r_{2}=100 \mathrm{~m}$, respectively, centered at the origin of a Cartesian coordinate system, as shown in Fig. 4. The speed limit of this road segment for the vehicle to keep is assumed to be $30 \mathrm{mi} / \mathrm{h}$ $(13.4 \mathrm{~m} / \mathrm{s})$.

Regarding the range and bearing measurement model in (27), it is assumed that a radar sensor is positioned at the origin. The corresponding measurement noise $v_{k}$ follows a Gaussian distribution with zero mean and covariance $R=$ $\operatorname{diag}\left\{36,10^{-2}\right\}$

$$
z_{k}=\left[\begin{array}{c}
\sqrt{x_{1, k^{+}}^{2} x_{2, k}^{2}} \\
\arctan \left(\frac{x_{2, k}}{x_{1, k}}\right)
\end{array}\right]+\boldsymbol{v}_{k} .
$$

Three algorithms are chosen for comparison for this simulated scenario including the EKF, the constrained

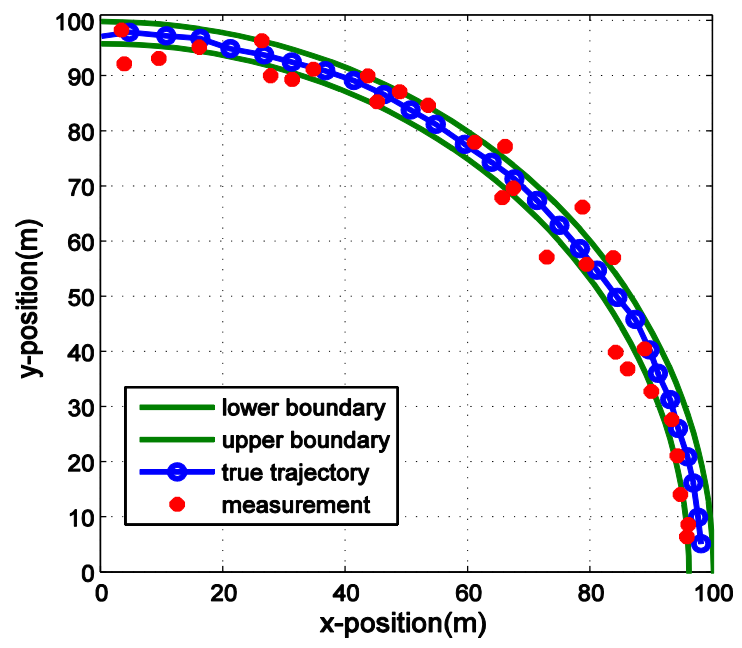

Fig. 4. Simulated circular road tracking scenario.

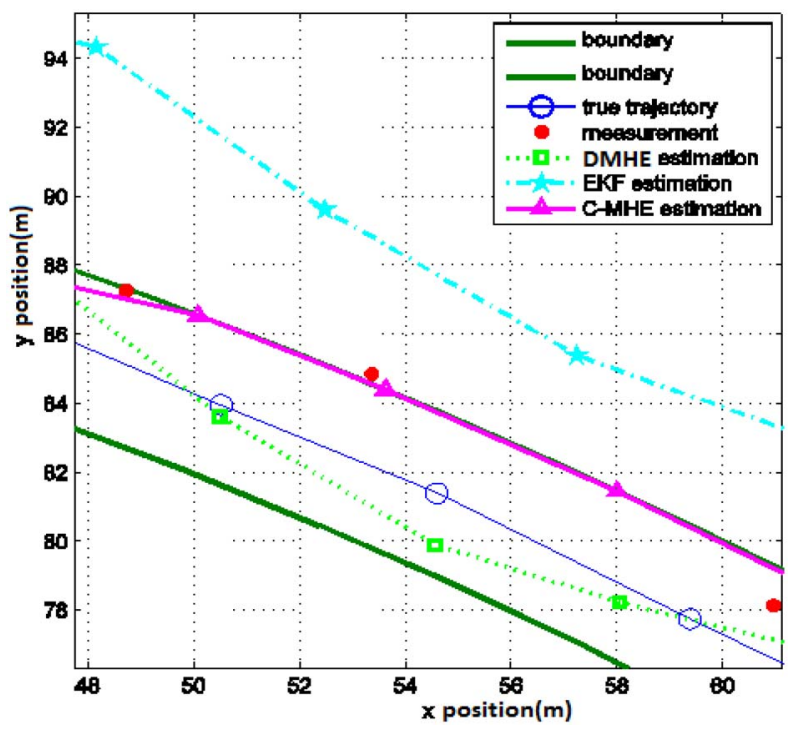

Fig. 5. True and estimated results for EKF, CMHE, and DMHE.

MHE (CMHE) which considers the road boundary constraint, and the proposed DMHE. The system dynamic model for tracking is the same as the previous scenario. The reason a constant velocity model is still used here is to emphasize the benefit of using domain knowledge in the target tracking even with a poor dynamic model. Although better nonlinear models (e.g., a constant turning model) could be used, by using a relatively less accurate dynamic model, the benefit of the additional force-based interaction information could be emphasized especially when comparing the DMHE with the CMHE. For the proposed DMHE method, additional interactions between the target and environment are considered by using two forces.

1) Road repulsive forces generated by the road upper and lower boundary.

2) Force acting in the opposite of movement tangential direction to prevent the vehicle from exceeding the speed limit. 


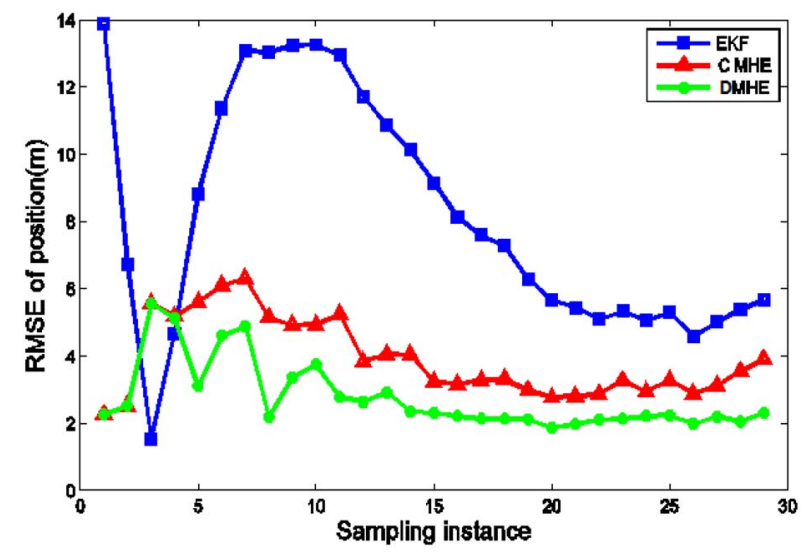

Fig. 6. RMSE of estimated position of EKF, CMHE, and DMHE.

TABLE III

AVERAged RMSES FOR POSITION USING EKF, CMHE, AND DMHE

\begin{tabular}{|c|c|c|c|}
\hline & EKF & CMHE & DMHE \\
\hline $\begin{array}{c}\text { RMSE } \\
(\mathrm{m})\end{array}$ & 8.8261 & 4.0494 & 2.9281 \\
\hline
\end{tabular}

For a fair comparison, all the algorithms are set to have the same initial condition with mean $\boldsymbol{x}_{0}=[75,0,10,10]^{T}$ and covariance $P_{0}=\operatorname{diag}\{10,10,1,1\}$.

First, a sample tracking performance of three different algorithms is illustrated in Fig. 5. It can be observed that the estimation result of the EKF is outside the road boundary. The performance is improved in the CMHE with the tracking results being projected on the road boundary. However, it is still quite different from the true trajectory. The most accurate and reasonable tracking result is obtained by the DMHE. Next, we perform numerical evaluations on three algorithms using the RMSEs through a hundred Monte Carlo simulations for the same scenario. Fig. 6 presents the averaged RMSE time history of the estimated position of each filter (the sampling interval is $0.5 \mathrm{~s}$ ). It can be seen that the DMHE approach achieves the minimum RMSEs during the majority of times. Besides, the averaged RMSEs for the whole target trajectory by different methods are presented in Table III. Again, the DMHE achieves the most accurate tracking performance. In comparison to the EKF and the CMHE, the averaged RMSE for position estimation by the DMHE is improved by $66.8 \%$ and $27.7 \%$, respectively.

\section{B. Multiple-Target Tracking}

1) Simulation Scenario: The performance of the DMHEMHT is compared against the EKF-MHT, and the CMHEMHT for MTT. Three vehicles are simulated to move in a realistic region (near Loughborough town in the U.K., and the region's geographic information is obtained from the GIS). As shown in Fig. 7, we consider a road intersection scenario with a rectangular region of surveillance, with an unknown and time varying number of targets observed in a cluttered environment. The vehicle dynamics is described the same

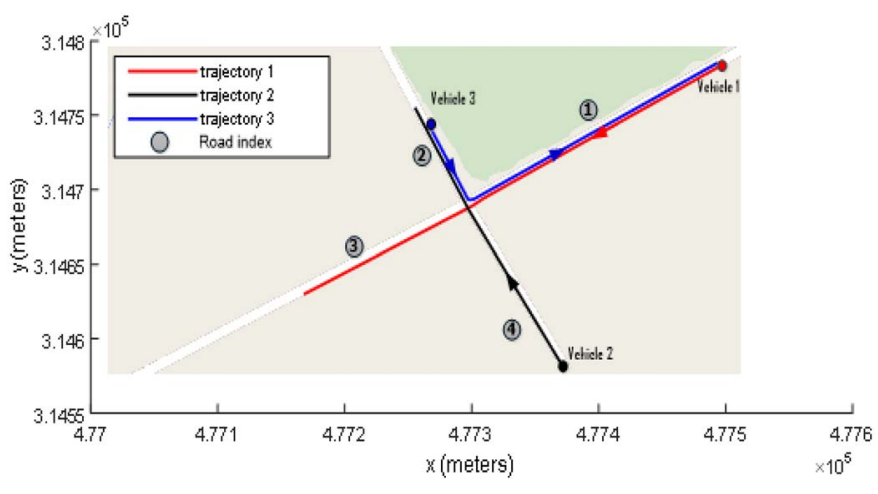

Fig. 7. MTT scenario.

as (21). The 2-D Gaussian process noise has covariance matrix $Q$ of $25 \mathrm{~m} / \mathrm{s}^{2}$. Initially, two targets start moving in the environment: 1) vehicle 1 (shown as the red point) heads to the southwest direction with an initial speed along road one of $12 \mathrm{~m} / \mathrm{s}$, it then crosses the intersection and travels on road 3 and 2) vehicle 2 (shown as the black point) starts from road 4 heading to the northwest direction with an initial speed along the road network of $8 \mathrm{~m} / \mathrm{s}$, it then crosses the intersection and travels on road 2. A new vehicle 3 starts to move 3 s later from road 2 with initial speed of $8 \mathrm{~m} / \mathrm{s}$ heading to southeast direction and then change its direction at the intersection heading to northeast on road 1. As shown in Fig. 7, tracking ambiguity occurs during the process around the intersection and on roads 1 and 2, which makes the problem challenging.

The target initial covariance is defined as $P_{0}=$ $\operatorname{diag}\{100,100,25,25\}$ for all three targets. Each target is detected with a probability of $P_{d}=0.98$. Regarding the range and bearing measurement model in (28), it is assumed that a radar sensor is positioned at the bottom right corner. The corresponding measurement noise $\boldsymbol{v}_{k}$ follows a Gaussian distribution with zero mean and covariance $R=\operatorname{diag}\left\{25,2.5^{-3}\right\}$. The detected measurements are immersed in a high clutter environment that can be modeled as a Poisson distribution with clutter density of $\beta_{\mathrm{FA}}=7.3 * 10^{-5}$ (false alarms/area/scan) over the $1.375 * 10^{5} \mathrm{~m}^{2}$ region (i.e., clutter returns over the region of interest).

2) Domain Knowledge Exploitation: The speed limitations of the main road (roads 1 and 3 along the east-west direction) and side road (roads 2 and 4 along the north-south direction) are $40 \mathrm{mi} / \mathrm{h}(17.9 \mathrm{~m} / \mathrm{s})$ and $30 \mathrm{mi} / \mathrm{h}(13.4 \mathrm{~m} / \mathrm{s})$, respectively. The road constraints are applied to constrain the vehicle positions and measurements. In addition to physical constraints, different target interactions with the environment are considered including interaction between: 1) the vehicle and road boundary; 2) the vehicle speed and speed limitation; and 3 ) vehicle in the minor road (2 and 4) and the junction (the vehicle in the minor road will slow down when it approaches the junction). Besides, the interactions between moving vehicles are also considered. These interactions are represented by forces, which is defined as

$$
\boldsymbol{f}_{i, j}^{\mathrm{rep} 4}= \begin{cases}A \cdot \exp \left(\frac{-d_{i j}}{B}\right) \boldsymbol{n}_{j i}, & \text { if } d_{i j} \leq D_{t} \\ 0, & \text { otherwise }\end{cases}
$$




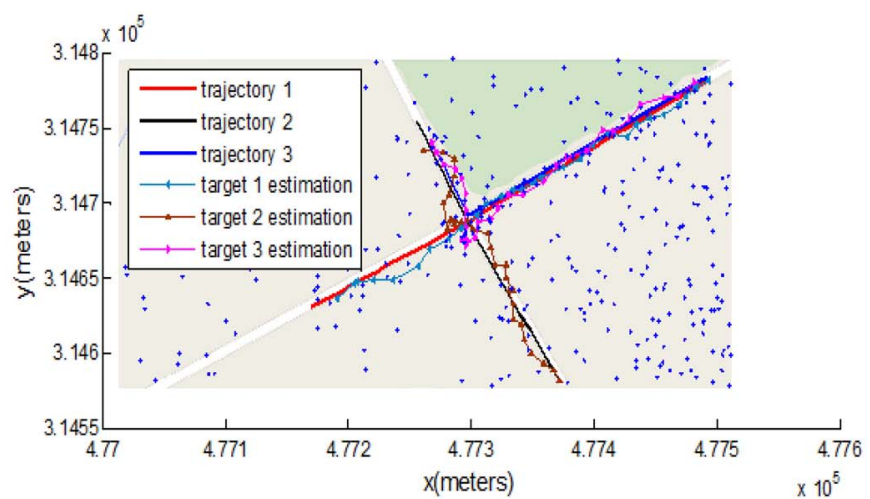

(a)

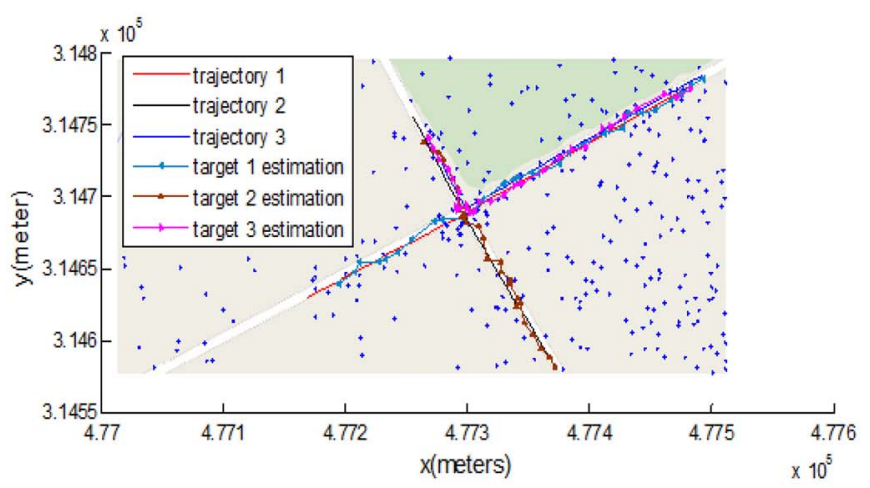

(b)

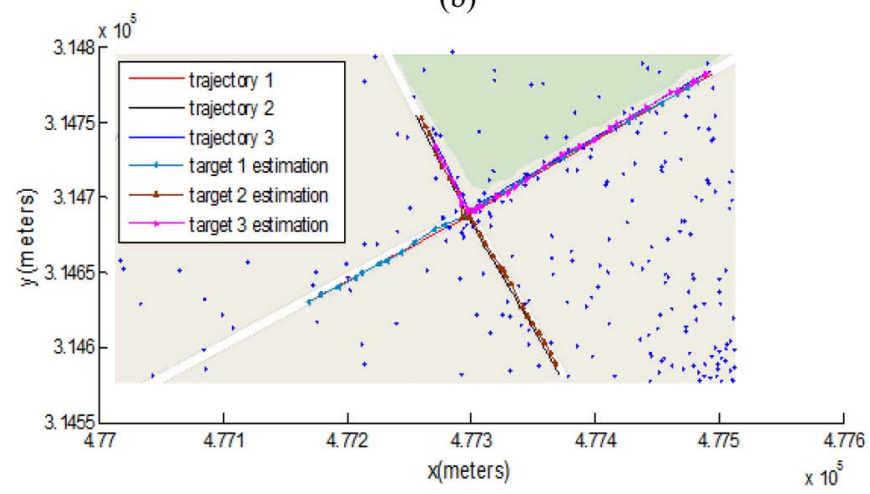

(c)

Fig. 8. (a) MTT using EKF-MHT. (b) CMHE-MHT with road constraint. (c) DMHE-MHT with force interaction model and road constraint. MTT scenario.

where $d_{i j}$ represents the relative distance between vehicles $i$ and $j$ in a Cartesian coordinate and $\mathrm{A}$ and $B$ are positive constant values. A threshold value $D_{t}$ is defined for interaction force so that repulsive behavior is activated only if the relative distance $d_{i j}$ is less than $D_{t}$.

3) Parameters Setting for the MHE and the MHT: The lifetime threshold is defined as 5 in the MHT implementation, which means any new target can only be confirmed if successfully detected in five consecutive time steps. Similarly, tracking any existing target will be terminated after miss detection of five sequential time steps. The horizon length used in the MHE is set as 4 and so as for $N$-scan pruning. Since only a small number of targets are considered in this paper, at each time step, three new hypotheses generated from one existing parent hypothesis are kept so as to reduce the computational cost.
TABLE IV

AVERAGED RMSES FOR THREE VEHICLES OF DIFFERENT APPROACHES

\begin{tabular}{|c|c|c|c|}
\hline & EKF-MHT & CMHE-MHT & DMHE-MHT \\
\hline $\begin{array}{c}\text { Overall RMSE } \\
\text { position (m) }\end{array}$ & 8.9004 & 5.6353 & 5.0077 \\
\hline $\begin{array}{c}\text { RMSE for } \\
\text { Target 1 (m) }\end{array}$ & 6.9271 & 5.4747 & 5.1271 \\
\hline $\begin{array}{c}\text { RMSE for } \\
\text { Target 2 (m) }\end{array}$ & 8.7000 & 5.3629 & 4.8760 \\
\hline $\begin{array}{c}\text { RMSE for } \\
\text { Target 3 (m) }\end{array}$ & 11.0740 & 6.0683 & 5.0200 \\
\hline
\end{tabular}

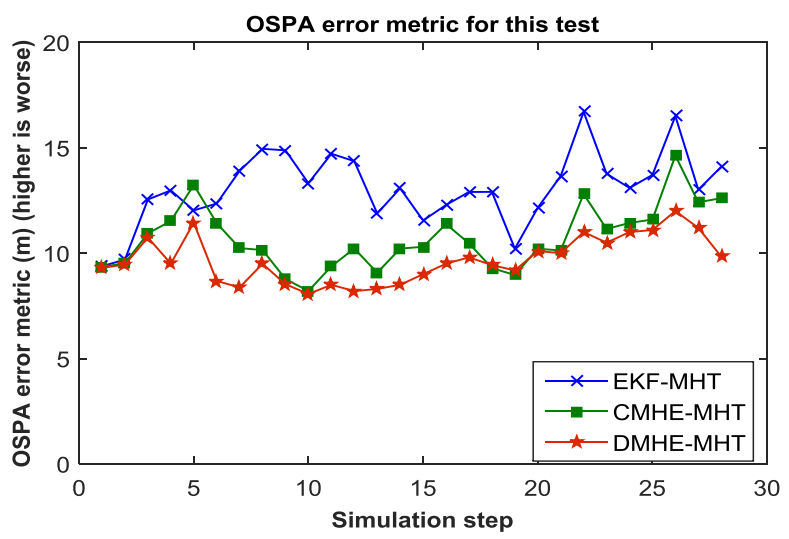

Fig. 9. OSPA for different algorithms.

The position estimates are shown in Fig. 8, and it can be shown that the road constraint and force based interaction play a significant part for improving the tracking accuracy. By comparing Fig. 8(a) and (b), we can find that map-based road boundary constraints improves the overall tracking results significantly. Due to the inequality state constraints, the vehicle positions are constrained within the road. The results get even better after introducing the force-based interaction information. In this case, the estimated vehicle trajectories are not only within the road boundaries but also get closer to the real trajectories. For further comparison between different algorithms, 50 trials of Monte Carlo simulations are performed. The performances of algorithms are measured using the RMSE. As shown in Table IV, the DMHE-MHT gives the best tracking results for all three targets by considering both road boundary constraint and force-based interaction. A more remarkable performance improvement is obtained for target 3 as it has the most interactions with the road and other incoming vehicles.

The MHT, the CMHE-MHT, and the proposed DMHT-MHE are also compared using the optimal subpattern assignment metric (OSPA) [27]. The OSPA is proposed for evaluating the performance of MTT algorithms, which considers not only the estimation performance but also association accuracy. The OSPA metric computes the distance between two sets of tracks by adding the error between target labels (or target indices) to the spatial distance. As can be seen in Fig. 9, the DMHE-MHT 
TABLE V

Averaged Computational Time of Different Approaches

\begin{tabular}{|c|c|c|c|}
\hline & EKF-MHT & CMHE-MHT & DMHE-MHT \\
\hline $\begin{array}{c}\text { Mean } \\
\text { computation time } \\
(\mathrm{s})\end{array}$ & 16.4650 & 25.4437 & 24.1360 \\
\hline
\end{tabular}

has the smallest OSPA value, which represents the smallest estimation error and least amount of incorrect data association. Besides, the proposed DMHE-MHT algorithm performance is more stable than the others by observing the variation of the OSPA distance over time, which presents the smoothest OSPA results.

The computation time of tracking algorithms is compared as shown in Table V. Each algorithm is run on a $2.4 \mathrm{GHz}$ PC for a hundred Monte Carlo simulations. The original MHT, using the EKF for state estimation considering no extra environmental information, shows the fastest computation time as expected. Comparing with the EKF, the MHE requires a higher computation cost due to the nature of optimization based on the quadratic programming. However, it still shows an acceptable computational load for a real time application. Note that the computational cost for the MHE heavily relies on the efficiency of the optimization method. In this paper, we use the optimization toolbox in the MATLAB software. There is only a slight computation time difference between the CMHE-MHT and the DMHE-MHT while the DMHE-MHT actually shows a better result. This is because after introducing the interaction information and using the improved MHT data association process, the relatively poor data association branches with a low probability are trimmed from the whole MHT hypotheses tree; thus less time is wasted on the unnecessary data association process.

\section{CONCLUSION}

This paper has proposed a new model-based ground vehicle tracking method considering domain knowledge in a comprehensive way. In particular, the physical road constraint together with a force-based dynamic model representing interactions between the target and the environment is used in the DMHE target tracking approach. This DMHE is further extended to the DMHE-MHT to deal with target association ambiguity, noisy measurements and multiple road model transition in MTT. By comparing the DMHE-based approach with traditional constrained state estimation methods using numerical simulation studies, it was shown that a significant improvement can be obtained in terms of target position estimate. Besides, the simulation results also showed that the proposed DMHE-MHT algorithm provides the most accurate tracking performance and robustness for an unknown and time varying number of targets observed in clutter environment using real road map constraint information and force-based target interaction information. To further verify the benefit and effectiveness of the proposed algorithm, real-world experiments with actual sensor measurements will be considered as future work.

\section{REFERENCES}

[1] M. S. Arulampalam, N. Gordon, M. Orton, and B. Ristic, "A variable structure multiple model particle filter for GMTI tracking," in Proc. 5th Int. Conf. Inf. Fusion, vol. 2. Annapolis, MD, USA, 2002, pp. 927-934.

[2] M. Ulmke and W. Koch, "Road-map assisted ground moving target tracking," IEEE Trans. Aerosp. Electron. Syst., vol. 42, no. 4, pp. 1264-1274, Oct. 2006.

[3] Y. Cheng and T. Singh, "Efficient particle filtering for road-constrained target tracking," IEEE Trans. Aerosp. Electron. Syst., vol. 43, no. 4, pp. 1454-1469, Oct. 2007.

[4] H. Oh, H. S. Shin, S. Kim, A. Tsourdos, and B. A. White, "Airborne behaviour monitoring using Gaussian processes with map information," IET Radar Sonar Navig., vol. 7, no. 4, pp. 393-400, Apr. 2013.

[5] M. Yu, C. Liu, W.-H. Chen, and J. Chambers, "A Bayesian framework with an auxiliary particle filter for GMTI-based ground vehicle tracking aided by domain knowledge," in Proc. SPIE Defense Security, Baltimore, MD, USA, 2014, Art. no. 90911I.

[6] C. Liu, B. Li, and W.-H. Chen, "Road network based vehicle navigation using an improved IMM particle filter," IFAC Proc. Vols., vol. 46, no. 10, pp. 193-198, 2013.

[7] D. Simon, "Kalman filtering with state constraints: A survey of linear and nonlinear algorithms,'IET Control Theory Appl., vol. 4, no. 8, pp. 1303-1318, Aug. 2010.

[8] O. Straka, J. Duník, and M. Šimandl, "Truncation nonlinear filters for state estimation with nonlinear inequality constraints," Automatica, vol. 48, no. 2, pp. 273-286, 2012.

[9] C. V. Rao, J. B. Rawlings, and D. Q. Mayne, "Constrained state estimation for nonlinear discrete-time systems: Stability and moving horizon approximations," IEEE Trans. Autom. Control, vol. 48, no. 2, pp. 246-258, Feb. 2003.

[10] X. R. Li and V. P. Jilkov, "Survey of maneuvering target tracking. Part I. Dynamic models," IEEE Trans. Aerosp. Electron. Syst., vol. 39, no. 4, pp. 1333-1364, Oct. 2003.

[11] T. Kirubarajan, Y. Bar-Shalom, K. R. Pattipati, and I. Kadar, "Ground target tracking with variable structure IMM estimator," IEEE Trans. Aerosp. Electron. Syst., vol. 36, no. 1, pp. 26-46, Jan. 2000.

[12] D. Helbing and P. Molnár, "Social force model for pedestrian dynamics," Phys. Rev. E, vol. 51, no. 5, p. 4282, 1995.

[13] G. Bang and I. S. Kweon, "Multi-target tracking using social force model in discrete-continuous optimisation framework," Electron. Lett., vol. 49, no. 21, pp. 1331-1333, Oct. 2013.

[14] R. Ding, M. Yu, and W. H. Chen, "A multiple target tracking strategy using moving horizon estimation approach," in Proc. 24th Int. Tech. Conf. Enhanc. Safety Veh. (ESV), Gothenburg, Sweden, 2015, pp. 1-13.

[15] X. R. Li and Y. Bar-Shalom, "Tracking in clutter with nearest neighbor filters: Analysis and performance," IEEE Trans. Aerosp. Electron. Syst., vol. 32, no. 3, pp. 995-1010, Jul. 1996.

[16] S. S. Blackrnan and R. Popoli, Design and Analysis of Modern Tracking Systems. Boston, MA, USA: Artech House, 1999.

[17] T. Fortmann, Y. Bar-Shalom, and M. Scheffe, "Sonar tracking of multiple targets using joint probabilistic data association," IEEE J. Ocean. Eng., vol. 8, no. 3, pp. 173-184, Jul. 1983.

[18] D. Reid, "An algorithm for tracking multiple targets," IEEE Trans. Autom. Control, vol. 24, no. 6, pp. 843-854, Dec. 1979.

[19] B.-N. Vo, S. Singh, and A. Doucet, "Sequential Monte Carlo methods for multitarget filtering with random finite sets," IEEE Trans. Aerosp. Electron. Syst., vol. 41, no. 4, pp. 1224-1245, Oct. 2005.

[20] S. S. Blackman, "Multiple hypothesis tracking for multiple target tracking," IEEE Aerosp. Electron. Syst. Mag., vol. 19, no. 1, pp. 5-18, Jan. 2004.

[21] D. Helbing, I. Farkas, and T. Vicsek, "Simulating dynamical features of escape panic," Nature, vol. 407, no. 6803, pp. 487-490, 2000.

[22] C. Yang, M. Bakich, and E. Blasch, "Nonlinear constrained tracking of targets on roads," in Proc. 7th Int. Conf. Inf. Fusion, vol. 1. Philadelphia, PA, USA, 2005, p. 8.

[23] S. Oh, S. Russell, and S. Sastry, "Markov chain Monte Carlo data association for general multiple-target tracking problems," in Proc. 43rd IEEE Conf. Decis. Control (CDC), vol. 1. Nassau, Bahamas, 2004, pp. 735-742.

[24] L. J. Cox and S. L. Hingorani, "An efficient implementation of Reid's multiple hypothesis tracking algorithm and its evaluation for the purpose of visual tracking," IEEE Trans. Pattern Anal. Mach. Intell., vol. 18, no. 2, pp. 138-150, Feb. 1996.

[25] D. Simon and T. L. Chia, "Kalman filtering with state equality constraints," IEEE Trans. Aerosp. Electron. Syst., vol. 38, no. 1, pp. 128-136, Jan. 2002. 
[26] H. A. P. Blom and E. A. Bloem, "Exact Bayesian and particle filtering of stochastic hybrid systems," IEEE Trans. Aerosp. Electron. Syst., vol. 43, no. 1, pp. 55-70, Jan. 2007.

[27] D. Schuhmacher, B.-T. Vo, and B.-N. Vo, "A consistent metric for performance evaluation of multi-object filters," IEEE Trans. Signal Process., vol. 56, no. 8, pp. 3447-3457, Aug. 2008.

[28] N. Bellomo and C. Dogbe, "On the modeling of traffic and crowds: A survey of models, speculations, and perspectives," SIAM Rev., vol. 53, no. 3, pp. 409-463, 2011.

[29] P. Kumar, R. Merzouki, B. Conrard, V. Coelen, and B. O. Bouamama, "Multilevel modeling of the traffic dynamic," IEEE Trans. Intell. Transp. Syst., vol. 15, no. 3, pp. 1066-1082, Jun. 2014.

[30] S. Pellegrini, A. Ess, K. Schindler, and L. van Gool, "You'll never walk alone: Modeling social behavior for multi-target tracking," in Proc. IEEE 12th Int. Conf. Comput. Vis., Kyoto, Japan, 2009, pp. 261-268.

[31] X. Shao, B. Huang, and J. M. Lee, "Constrained Bayesian state estimation-A comparative study and a new particle filter based approach," J. Process Control, vol. 20, no. 2, pp. 143-157, 2010.

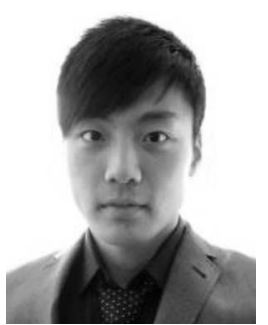

Runxiao Ding received the B.Eng. degree in automatic control and system engineering from the University of Sheffield, Sheffield, U.K., in 2012, and the Ph.D. degree in application of target tracking and path planning based techniques in autonomous vehicle field from Loughborough University, Loughborough, U.K., in 2016.

$\mathrm{He}$ is currently an Advanced Driver Assistance System (ADAS) Engineer with Jaguar Land Rover Limited, Coventry, U.K. His current research interests include autonomous vehicle field, such as ADASs, multiple-target tacking in situational awareness, path planning, decision making and control, estimation and sensor/information fusion, and vehicle modeling and testing.

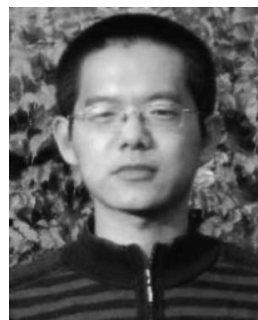

Miao Yu (M'15) was born in China, in 1986. He received the M.Sc. degree in digital communication system and the Ph.D. degree in the applications of computer vision based techniques for fall detection from Loughborough University, Loughborough, U.K., in 2008 and 2013, respectively.

$\mathrm{He}$ has been a Research Associate with the Department of Aeronautical and Automotive Engineering, Loughborough University since 2013. His current research interests include developing/implementing algorithms in statistical signal processing, machine learning and data/knowledge modeling, as well as the algorithms applications in the areas of object tracking, surveillance, abnormal detection and situation awareness in health care, and defence and intelligent transportation system.

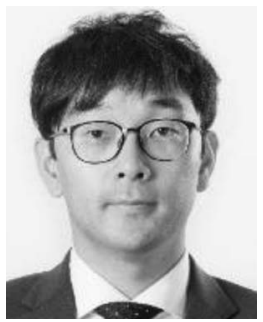

Hyondong Oh received the B.Sc. and M.Sc. degrees in aerospace engineering from the Korea Advanced Institute of Science and Technology, Daejeon, South Korea, in 2004 and 2010, respectively, and the $\mathrm{Ph} . \mathrm{D}$. degree in autonomous surveillance and target tracking guidance using multiple UAVs from Cranfield University, Cranfield, U.K., in 2013.

$\mathrm{He}$ was a Lecturer of Autonomous Unmanned Vehicles with Loughborough University, Loughborough, U.K., from 2014 to 2016 . He is currently an Assistant Professor with the School of Mechanical and Nuclear Engineering, Ulsan National Institute of Science and Technology, Ulsan, South Korea. His research interests include autonomy and decision making, cooperative control and path planning, nonlinear guidance and control, and estimation and sensor/information fusion for unmanned systems.

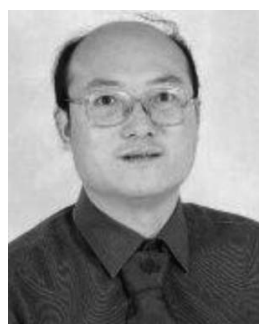

Wen-Hua Chen (M'00-SM'06) received the M.Sc. and Ph.D. degrees from Northeast University, Shenyang China, in 1989 and 1991, respectively.

$\mathrm{He}$ was a Lecturer and then an Associate Professor with the Department of Automatic Control, Nanjing University of Aeronautics and Astronautics, Nanjing, China, from 1991 to 1996. From 1997 to 2000, he held a research position and was then a Lecturer of Control Engineering with the Centre for Systems and Control, University of Glasgow, Glasgow, U.K. In 2000, he moved to the Department of Aeronautical and Automotive Engineering, Loughborough University, Loughborough, U.K., as a Lecturer, where he was appointed as a Professor in 2012. His current research interests include the development of unmanned autonomous intelligent systems and advanced control strategies, such as nonlinear model predictive control and disturbance observer based control, and their applications in aerospace and automotive engineering.

Dr. Chen is a Fellow of the Institution of Engineering and Technology and the Institution of Mechanical Engineers. 